\title{
EVALUACIÓN DE LA PERCEPCIÓN DE LA SONRISA EN PERSONAS CON Y SIN CONOCIMIENTOS EN ORTODONCIA
}

\author{
${ }^{1}$ Martha Cecilia Torres Llanes, ${ }^{1}$ Karla Barreto Rojas, ${ }^{1}$ Ángela María Polanco Carrillo, \\ ${ }^{2}$ Liliana Lucía Peña Pérez, ${ }^{3}$ Carlos Miguel Álvarez Rojas \\ ${ }^{1}$ Odontóloga U. Santo Tomás, estudiantes III año Especialización en Ortodoncia U. Santo Tomás, Colombia. \\ ${ }^{2}$ Odontóloga U. Nacional de Colombia, estudiante III año Especialización en Ortodoncia U. Santo Tomás, Colombia. \\ ${ }^{3}$ Máster en Ortodoncia y Ortopedia Maxilofacial CESO México, docente U. Santo Tomás, Colombia.
}

Autor responsable de correspondencia: Martha Cecilia Torres Llanes

Correo electrónico: marcetollan@hotmail.com

RESUMEN

Objetivo: evaluar la percepción de la sonrisa desde la perspectiva de los ortodoncistas y de las personas sin conocimientos de ortodoncia. Materiales y métodos: se realizó un estudio de corte transversal para lo cual se diligenciaron 207 cuestionarios de conocimientos y percepción de la sonrisa, por 43 ortodoncistas y estudiantes de la Especialización en Ortodoncia de la Universidad Santo Tomás (grupo 1) y 164 personas sin conocimientos de ortodoncia (grupo 2). Las variables de salida fueron la percepción de alteraciones en la línea media y en el corredor bucal; las variables explicatorias fueron la edad, el sexo y la condición socioeconómica. La información se describió mediante promedios y desviación estándar, y frecuencias y porcentajes según la naturaleza de las variables. Se aplicó la prueba de Chi cuadrado y se consideró un valor de $\mathrm{p}<0,05$ como estadísticamente significativo.

Resultados: la línea media con desviación de $1 \mathrm{~mm}$ y la centrada fueron las que más agradaron a hombres y mujeres, en los dos grupos de estudio. Al determinar la sonrisa menos agradable, los grupos coincidieron en que fue la de mayor desviación (4 mm). Se encontró asociación entre el conocimiento y la percepción de la desviación de la línea media en $2 \mathrm{~mm}(\mathrm{p}=0,044)$ y $4 \mathrm{~mm}(\mathrm{p}=0,000)$. Sonrisas con un corredor bucal amplio, medio y medio amplio son percibidas como menos agradables para ambos sexos, la totalidad de las mujeres valoró el corredor bucal amplio como menos agradable.

Conclusión: no se presentó una diferencia estadísticamente significativa en la percepción de las características de la sonrisa entre personas sin y con conocimientos de ortodoncia [Torres MC, Barreto K, Polanco AM, Peña LL, Álvarez CM. Evaluación de la percepción de la sonrisa en personas con y sin conocimientos en ortodoncia. Ustasalud. 2014; 13 : 32 - 39]

Palabras clave: sonrisa, ortodoncia, percepción.

\section{PERCEPTION OF THE SMILE ON PEOPLE WITH AND WITHOUT KNOWLEDGE IN ORTHODONTICS}

\section{ABSTRACT}

Objective: to assess the perception of the smile from the perspective of orthodontists and people without knowledge in orthodontics. Methods: a cross-sectional study was carried out. Two hundred and seven questionnaires about knowledge and perception of the smile were completed by 43 orthodontists and students of the Especialización en Ortodoncia at the Universidad Santo Tomás (group 1) and 164 people with no knowledge in orthodontics (group 2). The dependent variables were the perception of alterations in the midline and the buccal corridor; the explanatory variables were age, sex and socioeconomic status. The information was described by means and standard deviations, frequencies and percentages depending on the nature of the variables. Chi-square test was applied and a $\mathrm{p}$ value $<0.05$ was considered statistically significant.

Results: the midline deviation of $1 \mathrm{~mm}$ and centered were chosen for men and women as the most pleased the two study groups. In determining the least pleasant smile, the groups agreed that the least pleasant smile was when it presented the largest deviation (4 $\mathrm{mm})$. Association between knowledge and perception of the deviation from the mean line in $2 \mathrm{~mm}(\mathrm{p}=0.044)$ and $4 \mathrm{~mm}(\mathrm{p}=0.000)$ was found. Smiles with a large buccal corridor, medium and large were perceived as less pleasant for both sexes, all women assessed as less pleasant broad buccal corridor.

Conclusion: a statistically significant difference was not present in the perception of the characteristics of the smile between people with and without knowledge of orthodontics.

Key words: smiling, orthodontics, perception. 


\section{INTRODUCCIÓN}

Existen distintas percepciones de una sonrisa estéticamente aceptable entre ortodoncistas y personas sin conocimiento en ortodoncia, que se manifiesta en el distanciamiento entre los objetivos del tratamiento que busca el paciente y el profesional; mientras el profesional tarda meses en solucionar ciertos aspectos que para él son relevantes, el paciente no percibe la razón de la demora en su tratamiento y al finalizar este el paciente puede quedar insatisfecho, debido a que sus expectativas eran diferentes a las del profesional, por lo menos en cuanto a la percepción de su sonrisa. La importancia de una sonrisa atractiva ha variado con el pasar de los años y recientemente la evaluación de esta ha despertado un gran interés por parte de los clínicos, llevándolos a evaluar variables como el corredor bucal y la coincidencia de la línea media dental, a pesar de la poca evidencia científica existente. ${ }^{1-8}$

La evaluación del perfil facial en el diagnóstico ortodóncico ha tenido hasta ahora mayor relevancia que la evaluación de la sonrisa. ${ }^{8}$ Así por ejemplo, en el aniversario número 100 de la Sociedad Americana de Ortodoncia el logo fue "Ortodoncia 100 años de Sonrisa" y si se busca en Medline desde 1967 hasta 2008 con palabras clave como "Orthodontics" y "Smi$l e$ " se encuentran solo 23 referencias; por el contrario, si se busca "Orthodontics" y "Facial profile" se hallan 153 artículos. Entonces, parecería más apropiado que el eslogan fuera "Ortodoncia 100 años de Perfiles". ${ }^{9}$

El logro de estos objetivos estéticos presenta muchos retos que incluyen opiniones ocasionalmente divergentes entre pacientes y ortodoncistas en lo que constituye la estética de una sonrisa. ${ }^{10}$ Los estudios de estética de la sonrisa han sido complicados debido a la dificultad a la hora de crear modelos reales para el estudio de las variables de la sonrisa. ${ }^{4}$ Sarver y Akerman iniciaron la simulación digital para el análisis de la sonrisa y de esta manera poder identificar las diferentes percepciones de la sonrisa. ${ }^{9}$

En los últimos años, la sonrisa ha ganado una mayor importancia en cuanto a estética facial se refiere. El campo de la ortodoncia ha experimentado un "cambio de paradigma" para centrarse más en la estética, con especial énfasis en los tejidos blandos alrededor de la boca. ${ }^{11}$ Se ha descrito a la sonrisa como un importante método de influir en las personas, ${ }^{4}$ en la mente de las personas, la sonrisa ocupa el segundo lugar de importancia en cuanto al atractivo facial, el primero lo ocupan los ojos. ${ }^{5}$ Hoy día, gran parte de los ortodoncistas entienden que el logro de una óptima estética está relacionada no solo con una oclusión ideal sino que esta se caracterice por ser armónica facialmente. ${ }^{12}$ La comprensión de los factores que favorecen o desfavorecen el atractivo de una sonrisa es un paso importante en crear sonrisas atractivas. ${ }^{13}$

Muchos factores pueden influir en la formación de normas de belleza, como la cultura, los ingresos, y la edad. ${ }^{10}$ Esto implica que los ideales de belleza son siempre cambiantes. ${ }^{14,15}$ El rostro es considerado el aspecto más determinante de la apariencia física en las personas, la boca y los dientes se consideran fundamentales en la estética facial. ${ }^{16}$ Por lo tanto, es esencial controlar los efectos causados por el tratamiento ortodóncico mediante el conocimiento de los principios que proporcionan un equilibrio entre los dientes y los tejidos blandos durante una sonrisa. ${ }^{11,17,18}$ Es por esto que se requieren estudios sobre los detalles que influyen en la estética y el equilibrio entre los dientes y tejidos blandos. ${ }^{5}$

En algunos casos, unas sonrisas naturalmente atractivas junto con las sonrisas que son estéticamente agradables después del tratamiento dental no se ajustan a las normas de belleza, pero tampoco se consideran no atractivas. ${ }^{1,19}$ Los medios de comunicación visual y de entretenimiento han establecido estas normas estéticas para los espectadores al exponer caras hermosas y sonrisas brillantes. Esto ha tenido una influencia directa sobre cirugía cosmética y odontología.

Mediante este proyecto se pretendió identificar hasta dónde son perceptibles las desviaciones de la línea media por personas sin conocimiento en ortodoncia y conocer la preferencia en el ancho del corredor bucal para tenerlo en cuenta a la hora de la finalización de los tratamientos de ortodoncia y ahorrar así tiempo en la duración de los tratamientos, dinero y sobre todo tener pacientes satisfechos con los resultados de su sonrisa al finalizar la ortodoncia. Por lo tanto, el propósito de este estudio fue evaluar la percepción de la sonrisa desde la perspectiva de los ortodoncistas y de las personas sin conocimientos en ortodoncia.

\section{MATERIALES Y METODOS}

Se realizó un estudio observacional descriptivo de corte transversal. La población estuvo conformada por personas con y sin conocimiento en ortodoncia que hacen parte de la comunidad académica de la Universidad Santo Tomás en Floridablanca (Santander). La muestra fueron 207 personas, de las cuales 164 eran docentes vinculados con los diferentes programas que ofrece la Universidad Santo Tomás (sin conocimientos de ortodoncia) y 43 docentes y estudiantes de IV y VI semestre de la Especialización en Ortodoncia (con conocimiento en ortodoncia) de 
la misma institución. El grupo con conocimiento se denominó Grupo 1 y el grupo sin conocimiento Grupo 2.

Dentro de los criterios de inclusión se tuvieron en cuenta adultos jóvenes con título universitario o especialización que mostraran seriedad e interés al participar en el estudio. Se evaluó la percepción de alteraciones en la línea media, la percepción de alteraciones en el corredor bucal, la edad, el sexo y la condición socioeconómica.

\section{Procedimiento}

Se tomaron una serie de fotografias de sonrisa para poder tener una fotografia inicial y a partir de esta realizar las modificaciones necesarias para crear el formato de evaluación. Esta fotografia contó con los parámetros de una sonrisa ideal y dentro de estos la línea media dental centrada con respecto a la facial y un corredor bucal estrecho. Para la elección de la persona a la cual se le iba a tomar la fotografia, se realizó un examen clínico de varias mujeres que no habían recibido tratamiento de ortodoncia y que presentaban una sonrisa agradable. Luego de analizar la sonrisa por parte de los examinadores y de haber confirmado el cumplimiento de los parámetros ideales, como por ejemplo alineación dental, línea media dental centrada, corredor bucal estrecho, color dental, margen gingival, sonrisa dental entre otros se escogió la mejor.

La toma de fotografias se realizó con una cámara digital marca Canon EOS Rebel XT, de 8 mega pixeles con un macro 100, diafragma en 13 y velocidad en 100. La toma de la fotografía se realizó con una sonrisa fingida, la paciente fue ubicada en un cefalostato para reproducir la posición natural de la cabeza y se ubicó el lente de la cámara perpendicular a la posición natural de la cabeza. La fotografia fue tomada por una persona experta en fotos intraorales. Se hicieron varias tomas de la sonrisa que fueron valoradas por el equipo investigador y por el profesional en Adobe PhotoShop 7.0 para elegir la que presentara mejor color, resolución y las mejores características, para ser manipulada. La fotografía elegida se procesó por el experto en el programa Adobe PhotoShop 7.0 para alterar la línea media dental y el corredor bucal. Además, se realizaron retoques estéticos con el fin de dar una imagen más natural.

Alteraciones de la línea media: se alteró la línea media dental a $1 \mathrm{~mm}, 2 \mathrm{~mm}, 3 \mathrm{~mm}$ y $4 \mathrm{~mm}$ (según las evaluaciones de Kokich y colaboradores) hacia la izquierda al tomar como referencia el filtrum del labio superior y evaluar si el fenómeno de percepción de desviación de la línea media era igual para la población por estudiar.
Alteraciones en el corredor bucal: para realizar las variaciones del corredor bucal se utilizó la siguiente fórmula:

Sonrisa total: $\frac{\text { ancho visible dientes maxilares }}{\text { ancho de la comisura interna }}$

diferencia del ancho de dientes maxilares y ancho de la comi-

Corredor Bucal: sura interna

ancho de la comisura interna

Estos datos se dieron en porcentajes y la medida inicial fue del 100\%. Según esto, se realizó la modificación para el corredor bucal de la siguiente manera: $28 \%$ estrecho, $22 \%$ medio estrecho, $15 \%$ medio, $10 \%$ medio amplio, $2 \%$ amplio. $^{3}$

Presentación de las fotografias: las fotografias fueron impresas en una hoja tamaño carta por grupos, en cada hoja fueron presentadas cinco fotografias ordenadas al azar verticalmente en papel fotográfico y se les entregaron a los encuestados en dos grupos: uno con alteraciones de línea media y otro con alteraciones de corredor bucal. Las fotografias se enumeraron de 1 a 5 para ser evaluadas. El tiempo de evaluación de cada grupo de fotografías fue de aproximadamente cinco minutos y la encuesta fue auto administrada, pero en algunos casos los entrevistados necesitaron asesoría (Figuras 1 y 2).

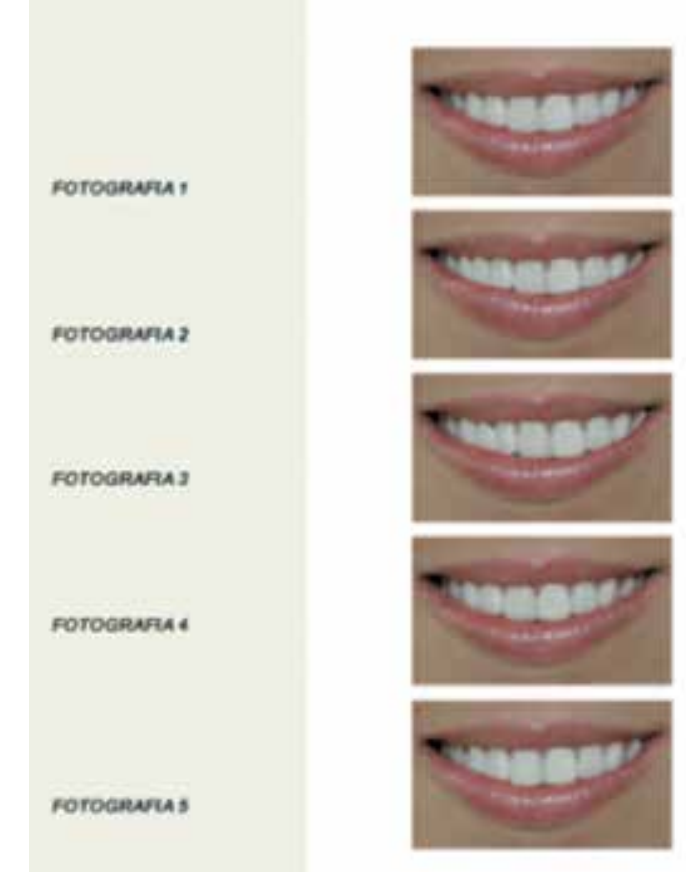

Figura 1. Presentación de las fotografias para evaluar la línea media dental 


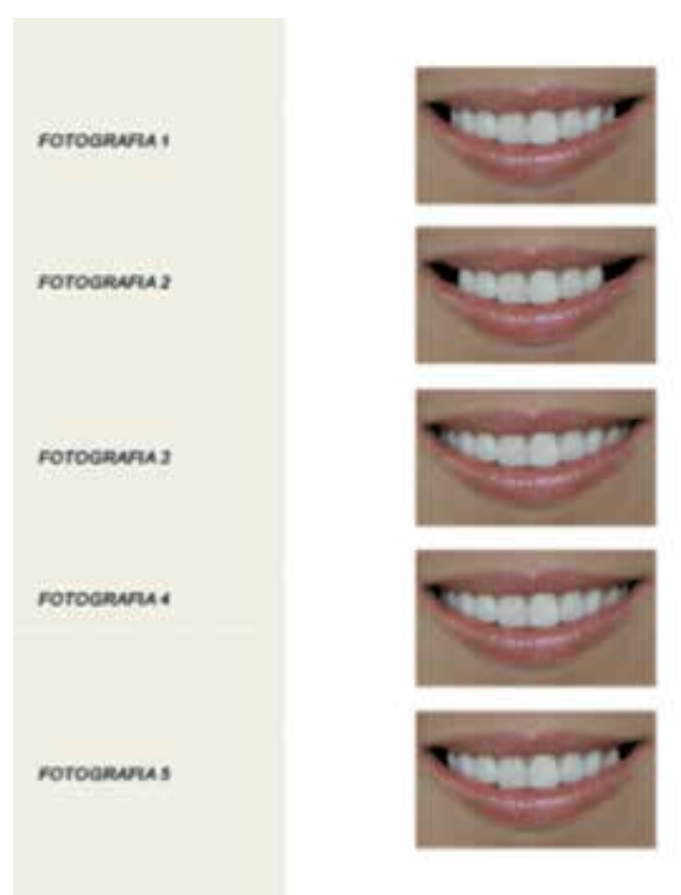

Figura 2. Presentación de las fotografias para evaluar el corredor bucal

Evaluación de las fotografias: a los participantes del estudio se les entregó un formato para la evaluación de las fotografias de la sonrisa, en el que evaluaron cada una de las fotografias como menos agradable y agradable. En el momento de la recolección de información se consideró que el docente o estudiante estuviera en un lugar iluminado en un escritorio cómodo y con mínimo diez minutos de disponibilidad para diligenciar el cuestionario.

\section{Análisis estadístico}

Se realizó una descripción univariada de las características de estudio mediante promedios y desviación estándar, frecuencias y porcentajes según la naturaleza de las variables. Un análisis bivariado fue realizado entre las variables dependientes (percepción de línea media y percepción de corredor bucal) y las variables independientes (grupos de edad, sexo, condición socioeconómica y grupos con y sin conocimiento en odontología). Para el análisis estadístico se empleó el paquete estadístico SPSS versión 15, se aplicó la prueba Chi cuadrado con una significancia estadística de $\mathrm{p}<0,05$.

\section{RESULTADOS}

Se diligenciaron 207 cuestionarios, 43 del grupo con conocimientos en ortodoncia, denominado Grupo 1, de los cuales $14(32,6 \%)$ eran hombres y 164 participantes sin conocimientos en ortodoncia, denominado Grupo 2, de los cuales $96(58,54 \%)$ fueron hombres.
En el Grupo 1, el promedio de edad fue 33 años y el estrato socioeconómico más frecuente fue 4. La percepción de desviación de línea media fue a partir de $2 \mathrm{~mm}$ al considerar una desviación de 1 $\mathrm{mm}$ dentro de una sonrisa atractiva, la percepción del corredor bucal tomada como agradable fue el corredor bucal amplio y el menos agradable el medio estrecho y estrecho.

En el Grupo 2, el promedio de edad fue 39 años y el estrato socioeconómico más frecuente fue 4 . Este grupo percibió como más atractiva la sonrisa con una desviación de $3 \mathrm{~mm}$ y de $1 \mathrm{~mm}$, y consideró menos atractiva una desviación de $4 \mathrm{~mm}$; con respecto al corredor bucal, se percibió mayor atractivo una sonrisa con corredor bucal ni ancho ni angosto.

En la Tabla 1 se observa la percepción de la sonrisa cuando varía la línea media y los resultados se presentan por sexo y grupo de estudio. La línea media con desviación de $1 \mathrm{~mm}$ y la centrada son las que más agradaron a hombres y a mujeres en los dos grupos de estudio, en promedio el 83\% lo consideraron así. Al determinar la sonrisa menos agradable, nuevamente, tanto hombres como mujeres en los dos grupos coincidieron en que era aquella que presentaba mayor desviación (4 mm).

A pesar de que las opiniones coincidieron en el caso de la percepción de la línea media en la sonrisa más agradable y la menos agradable, vale la pena resaltar que en las desviaciones de $2 \mathrm{~mm}$ y $3 \mathrm{~mm}$, la valoración por parte de los hombres y las mujeres cambia. Por ejemplo, en el grupo con conocimiento un $75,86 \%$ de las mujeres consideraron que una sonrisa con una línea media desviada $3 \mathrm{~mm}$ es menos agradable, cosa que no sucedió con los hombres en ninguno de los dos casos cuyos porcentajes de agrado y menos agrado son similares (Tabla 1).

La Tabla 2 describe los resultados de la percepción de la sonrisa al variar el corredor bucal de acuerdo con el sexo y el grupo de estudio. Según los resultados, las percepciones para hombres y mujeres coincidieron en los diferentes corredores bucales, en los dos grupos de estudio. La sonrisa con corredor bucal estrecho o medio estrecho fue considerada como agradable, tanto para hombres como para mujeres, aunque los hombres con conocimiento la percibieron mucho mejor $(92,86 \%)$. Sonrisas con corredores bucales amplio, medio y medio amplio son percibidas como menos agradables para ambos sexos, la totalidad de las mujeres valoró el corredor bucal amplio como menos agradable y los hombres estuvieron muy cerca de esta valoración con el 92,86\%. 


\section{ARTÍCULO DE INVESTIGACIÓN CIENTÍFICA Y TECNOLÓGICA}

La Tabla 3 presenta la relación de los parámetros ideales de la sonrisa (línea media centrada y corredor bucal estrecho) con las variables independientes. Se observó una relación estadísticamente significativa al evaluar el conocimiento con la percepción de la línea media centrada $(\mathrm{p}=0,048)$. Así mismo, se encontró una relación entre el conocimiento y la percepción de la desviación de la línea media en 2 $\mathrm{mm}(\mathrm{p}=0,044)$ y $4 \mathrm{~mm}(\mathrm{p}=0,000)$.

Tabla 1. Percepción de la línea media por sexo según el grupo de estudio

\begin{tabular}{|c|c|c|c|}
\hline Grupo & Fotografia & $\begin{array}{c}\text { Hombres } \\
\mathbf{n}(\%)\end{array}$ & $\begin{array}{c}\text { Mujeres } \\
\text { n (\%) }\end{array}$ \\
\hline \multirow{12}{*}{ Con conocimi } & Desviada $1 \mathrm{~mm}$ & & \\
\hline & Menos agradable & $3(21,43)$ & $4(13,79)$ \\
\hline & Agradable & $11(78,57)$ & $25(86,21)$ \\
\hline & Desviada $3 \mathrm{~mm}$ & & \\
\hline & Menos agradable & $6(42,86)$ & $22(75,86)$ \\
\hline & Agradable & $8(57,14)$ & $7(24,14)$ \\
\hline & Desviada 4 mm & & \\
\hline & Menos agradable & $12(85,71)$ & $27(93,10)$ \\
\hline & Agradable & $2(14,29)$ & $2(6,90)$ \\
\hline & Centrada & & \\
\hline & Menos agradable & $5(35,71)$ & $12(41,38)$ \\
\hline & Agradable & $9(64,29)$ & $17(58,62)$ \\
\hline \multirow{12}{*}{ Sin conocimi } & Desviada $1 \mathrm{~mm}$ & & \\
\hline & Menos agradable & $20(20,83)$ & $9(13,24)$ \\
\hline & Agradable & $76(79,17)$ & $59(86,76)$ \\
\hline & Desviada $3 \mathrm{~mm}$ & & \\
\hline & Menos agradable & $49(51,04)$ & $33(48,53)$ \\
\hline & Agradable & $47(48,96)$ & $35(51,47)$ \\
\hline & Desviada $4 \mathrm{~mm}$ & & \\
\hline & Menos agradable & $64(66,67)$ & $38(55,88)$ \\
\hline & Agradable & $32(33,33)$ & $30(44,12)$ \\
\hline & Centrada & & \\
\hline & Menos agradable & $21(21,88)$ & $19(27,94)$ \\
\hline & Agradable & $75(78,13)$ & $49(72,06)$ \\
\hline
\end{tabular}




\section{ARTÍCULO DE INVESTIGACIÓN CIENTÎFICA Y TECNOLÓGICA}

Tabla 2. Percepción del corredor bucal por sexo según el grupo de estudio

\begin{tabular}{|c|c|c|c|}
\hline Grupo & Fotografia & $\begin{array}{c}\text { Hombres } \\
\text { n (\%) }\end{array}$ & $\begin{array}{c}\text { Mujeres } \\
\text { n (\%) }\end{array}$ \\
\hline \multirow{15}{*}{ Con conocimientos } & Medio & & \\
\hline & Menos agradable & $14(100,0)$ & $29(100,0)$ \\
\hline & Agradable & -- & -- \\
\hline & Amplio & & \\
\hline & Menos agradable & $13(92,86)$ & $29(100,0)$ \\
\hline & Agradable & $1(7,14)$ & -- \\
\hline & Estrecho & & \\
\hline & Menos agradable & $1(7,14)$ & $9(31,03)$ \\
\hline & Agradable & $13(92,86)$ & $20(68,97)$ \\
\hline & Medio estrecho & & \\
\hline & Menos agradable & $2(14,29)$ & $7(24,14)$ \\
\hline & Agradable & $12(85,71)$ & $22(75,86)$ \\
\hline & Medio amplio & & \\
\hline & Menos agradable & $14(100,0)$ & $27(93,10)$ \\
\hline & Agradable & -- & $2(6,90)$ \\
\hline \multirow{15}{*}{ Sin conocimientos } & Medio & & \\
\hline & Menos agradable & $77(80,21)$ & $48(70,59)$ \\
\hline & Agradable & $19(19,79)$ & $20(29,41)$ \\
\hline & Amplio & & \\
\hline & Menos agradable & $78(81,25)$ & $52(76,47)$ \\
\hline & Agradable & $18(18,75)$ & $16(23,53)$ \\
\hline & Estrecho & & \\
\hline & Menos agradable & $12(12,50)$ & $13(19,12)$ \\
\hline & Agradable & $84(87,50)$ & $55(80,88)$ \\
\hline & Medio estrecho & & \\
\hline & Menos agradable & $22(22,92)$ & $10(14,71)$ \\
\hline & Agradable & $74(77,08)$ & $58(85,29)$ \\
\hline & Medio amplio & & \\
\hline & Menos agradable & $66(68,75)$ & $46(67,55)$ \\
\hline & Agradable & $30(31,25)$ & $22(32,35)$ \\
\hline
\end{tabular}


Tabla 3. Asociación entre los parámetros ideales de la sonrisa y las variables independientes

\begin{tabular}{lccc}
\hline \multicolumn{1}{c}{ Línea media } & $\begin{array}{c}\text { Menos agradable } \\
\text { n (\%) }\end{array}$ & $\begin{array}{c}\text { Agradable } \\
\text { n (\%) }\end{array}$ & $\mathbf{P}^{*}$ \\
\hline Desviada 1 mm & & & 0,829 \\
Con conocimiento & $7(16,3)$ & $36(83,7)$ & \\
Sin conocimiento & $29(17,7)$ & $135(82,3)$ & \\
Desviada 3 mm & & & 0,077 \\
Con conocimiento & $28(65,1)$ & $15(34,9)$ & \\
Sin conocimiento & $82(50,0)$ & $82(50,0)$ & 0,000 \\
Desviada 4 mm & & & \\
Con conocimiento & $39(90,7)$ & $4(9,3)$ & 0,048 \\
Sin conocimiento & $102(62,2)$ & $62(37,8)$ & \\
Centrada & & & \\
Con conocimiento & $17(39,5)$ & $26(60,5)$ & \\
Sin conocimiento & $40(24,4)$ & $124(75,6)$ & \\
Desviada 2 mm & & $20(46,5)$ & \\
Con conocimiento & $23(53,5)$ & $104(63,4)$ & \\
Sin conocimiento & $60(36,6)$ & & \\
\hline cuadrado.
\end{tabular}

\section{DISCUSIÓN}

Al evaluar el tipo de percepción en el grupo de personas con y sin conocimiento en ortodoncia, en cuanto a la desviación de la línea media se observó que la desviación de $1 \mathrm{~mm}$ y la centrada fue la de mayor agrado para los dos grupos, así también coincidieron en afirmar que la desviación de $4 \mathrm{~mm}$ era la de menor agrado $(p>0,05)$. Datos contrarios fueron reportados en el estudio de Kokichy colaboradores (1999) quienes encontraron que solo los ortodoncistas tenían la habilidad de identificar la desviación de la línea media dental; ${ }^{6}$ los odontólogos generales y personas sin conocimiento en odontología no encontraron diferencias en la percepción de la línea media incluso cuando la desviación era de $4 \mathrm{~mm}$.

Posteriormente en 2006, Kockich y colaboradores al evaluar asimetrías de la estética dental encontraron que estas alteraciones son menos atractivas para los profesionales de odontología y personas del común. ${ }^{5}$ Así mismo, Jhonston, Burden y Stevenson encontraron en su estudio que los ortodoncistas son más sensibles al detectar discrepancias de línea media, comparado con personas del común y que estas últimas pueden llegar a percibir como menos atractivo desviaciones de la línea media mayor a $2 \mathrm{~mm} .{ }^{20}$

No se observó una diferencia estadísticamente significativa al relacionar el sexo en cada grupo. Este hallazgo no coincide con los encontrados por Rodríguez y colabores quienes sí observaron diferencias en la percepción de la línea media entre la edad categorizada y el sexo. ${ }^{14}$ Por otra parte, Zhang y colaboradores (2010) reportaron que las mujeres fueron menos tolerantes ante la desviación de línea media era más notable en personas de caras largas característica que no aplica a este estudio debido a que solo se utilizó la región de la sonrisa. ${ }^{12}$

Las preferencias del corredor bucal para personas con y sin conocimiento de ortodoncia fueron estrecho y medio estrecho, resultados similares a los estudios publicados por Moore y col, Ioi y colaboradores, Martin y colaboradores, y Parekh y colaboradores. ${ }^{1,3,19,21}$ Por el contrario, Ritter y colaboradores no hallaron mayor influencia en la estética de la sonrisa ni diferencia en la evaluación entre ortodoncista y personas del común en relación con un corredor bucal amplio. ${ }^{22}$

Cabe señalar que los datos proporcionados en el estudio son el resultado del análisis de la fotografia solo de la región de sonrisa y no de una fotografia de cara completa. Es necesario tener en cuenta esta apreciación puesto que la apariencia facial del paciente podría ser importante a la hora de evaluar la sonrisa. Sin embargo, Havens y colaboradores sugirieron que la estética de la cara no depende de características faciales aisladas. ${ }^{10}$

El análisis de los parámetros de línea media y corredor bucal fueron evaluados en una forma estática mediante la valoración de fotografias de sonrisa y 
no en una forma dinámica como lo proponen Maulik y Nanda. Estos autores realizaron un análisis dinámico de la sonrisa y reportaron que la inclinación de la cabeza y la velocidad al sonreír pueden generar percepciones diferentes de la sonrisa. ${ }^{23}$

Adicionalmente, Kockich y colaboradores realizaron una prueba de personalidad a los grupos evaluadores de línea media sin encontrar una diferencia estadísticamente significativa, ${ }^{5,6}$ razón por la cual esta prueba no se tuvo en cuenta en el presente estudio. Tampoco fueron contempladas las alteraciones visuales de los evaluadores, lo cual podría alterar la percepción a la hora de evaluar los diferentes parámetros de la sonrisa.

\section{Conclusiones}

El sexo no es una variable que afecte la percepción de la sonrisa cuando varía la línea media.

La edad parece no afectar la percepción de la línea media y el corredor bucal a excepción de personas mayores de 60 años.

No hubo una diferencia estadísticamente significativa entre las percepciones de línea media y el corredor bucal de las personas con y sin conocimientos de ortodoncia.

El ancho del corredor bucal influyó en la percepción que las personas tienen de una sonrisa, entre más amplio sea el corredor menos agradable.

\section{BIBLIOGRAFÍA}

1. Moore T, Southard KA, Casko JS, Qian F, Southard TE. Buccal corridors and smile esthetics. Am J Orthod Dentofacial Orthop. 2005; 127: 208-213.

2. Van der Geld P, Oosterveld P, Van Heck G, Kuijpers-Jagtman AM. Smile attractiveness. Self-perception and influence on personality. Angle Orthod. 2007; 5: 759-765.

3. Ioi H, Nakata S, Counts AL. Effects of buccal corridors on smile esthetics in Japanese. Angle Orthod. 2009; 79: 628633.

4. Parekh SM, Fields HW, Beck M, Rosenstiel S. Attractiveness of variations in the smile arc and buccal corridor space as judged by orthodontists and laymen. Angle Orthod. 2006; 76: 557-563.

5. Kokich VO, Kokich VG, Kiyak HA. Perceptions of dental professionals and laypersons to altered dental esthetics: Asymmetric and symmetric situations. Am J Orthod Dentofacial Orthop. 2006; 130: 141-151.

6. Kokich VO, Kiyak HA, Shapiro PA. Comparing the perception of dentists and lay people to altered dental esthetics. J Esthet Dent. 1999; 11: 311-324.

7. Brislin RW, Lewis SA. Dating and physical attractiveness: replication. Psychol Rep. 1968; 22: 976.

8. Boley JC, Pontier JP. Facial change in extraction and nonextraction in patients. Angle Orthod. 1998; 68: 539-546.

9. Sarver P, Ackerman M. Dynamic smile visualization and quantification: Part 1. Evolution of the concept and dy- namic records for smile capture. Am J Orthod Dentofacial Orthop. 2003; 124: 4-12.

10. Havens DC, McNamara JA Jr, Sigler LM, Baccetti T. The role of the posed smile in overall facial esthetics. Angle Orthod. 2010; 80: 322-328.

11. McNamara L, McNamara JA Jr, Ackerman MB, Baccetti T. Hard - and soft tissue contributions to the esthetics of the posed smile in growing patients seeking orthodontic treatment. Am J Orthod Dentofacial Orthop. 2008; 133: 491-499.

12. Zhang YF, Xiao L, Li J, Peng YR, Zhao Z. Young people's esthetic perception of dental midline deviation. Angle Orthod. 2010; 80: 515-520.

13. Tjan AH, Miller GD, The JG. Some esthetics factors in a smile. J Prosthet Dent. 1984; 51: 24-28.

14. Rodríguez C de D, Magnani R, Machado MS, Oliveira OB. The perception of smile attractiveness, variations from esthetic norms, photographic framing and order of presentation. Angle Orthod. 2009; 79: 634-639.

15. Singer BA. Principles of esthetics. Curr Opin Cosmet Dent. 1994: 6-12.

16. Rufenacht C. Fundamentals of Esthetics. Quintessense Books. Pag 11-132.

17. Hulsey CM. An esthetic evaluation of lip-teeth relationships present in the smile. Am J Orthod Dentofacial Orthop. 1970; 57: 132-144.

18. Peck S, Peck L, Katoja M. Some vertical lineaments of lip position. Am J Orthod Dentofacial Orthop. 1992; 101: 519-524.

19. Martin AJ, Buschang PH, Boley JC, Taylor RW, McKinney TW. The impact of buccal corridors on smile attractiveness. Eur J Orthod. 2007; 29: 530-537.

20. Johnston CD, Burden DJ, Stevenson MR. The influence of dental to facial midline discrepancies on dental attractiveness rating. Eur J Orthod. 1999; 21: 517-522.

21. Parekh S, Fields HW, Beck FM, Rosenstiel SF. The acceptability of variations in smile arc and buccal corridor space. Orthod Craniofacial Res. 2007; 10: 15-21.

22. Ritter DE, Gandini LG, Pinto Ados S, Locks A. Esthetic influence of negative space in the buccal corridor during smiling. Angle Orthod. 2006; 76: 198-203.

23. Maulik C, Nanda R. Dynamic smile analysis in young adults. Christopher Maulik and Ravindra Nanda. Am J Orthod Dentofacial Orthop. 2007; 132: 307-315.

\section{Correo electrónico de los autores:}

Martha Cecilia Torres Llanes: marcetollan@hotmail.com Carlos Miguel Álvarez Rojas: camialro@hotmail.com

Karla Barreto Rojas: karlabarretorojas15@hotmail.com

Liliana Lucía Peña Pérez: lilianaluciapp@hotmail.com Ángela María Polanco Carrillo: angelamaria0711@hotmail.com 